\title{
Moscas ectoparasitas (Diptera, Streblidae e Nycteribiidae) em morcegos (Mammalia, Chiroptera) em área de Floresta com Araucária no Estado do Paraná, sul do Brasil
}

\author{
Gustavo Graciolli ${ }^{1}$ \& Gledson V. Bianconi ${ }^{2}$ \\ ${ }^{1}$ Departamento de Biologia, Centro de Ciências Biológicas e da Saúde, Universidade Federal de Mato Grosso do Sul. \\ Caixa Postal 549, 79070-900 Campo Grande, Mato Grosso do Sul, Brasil. E-mail: ggraciolli@yahoo.com.br \\ 2 Programa de Pós-graduação em Ciências Biológicas, Zoologia, Universidade Estadual Paulista. Caixa Postal 199, \\ 13506-900 Rio Claro, São Paulo, Brasil. E-mail: bianconi@terra.com.br
}

\begin{abstract}
Ectoparasitic bat flies (Diptera, Streblidae e Nycteribiidae) on bats (Mammalia, Chiroptera) of the Araucaria Forest, State of Paraná, South Brazil. The information available on streblid and nycteribiid bat flies and their hosts are scant in the Araucaria Forest (AF) and there are no data on richness, distribution patterns or abundance of these ectoparasites in bat communities. Therefore, we conducted a study on bats and their ectoparasites in AF remnants (approximately 470 ha) located in the municipality of Fazenda Rio Grande, Paraná State, between December 2001 and May 2003. Twelve individuals of three species of Nycteribiidae and 119 individuals of six species of Streblidae were collected on 127 bats (four species of Phyllostomidae and seven of Vespertilionidae). Five of the six species of streblid were found exclusively on Sturnira lilium (E. Geoffroy, 1810) and Artibeus lituratus (Olfers, 1818). We collected Megistopoda proxima (Séguy, 1926) on 62 S. lilium examined with prevalence of $65.4 \%$ and mean intensity of 2.00; Aspidoptera falcata Wenzel, 1976, respectively, with $13.5 \%$ and 2.29; Trichobius phyllostomae Kessel, 1925 with $7.7 \%$ and 1.00; and Metelasmus wenzeli Graciolli \& Dick, 2004 with $1.6 \%$ and 4.00 . We did not find significant differences in prevalence $(\mathrm{p}=0.544)$ and mean intensity $(\mathrm{p}=0.361)$ between the sexes of $S$. lilium. On $26 \mathrm{~A}$. lituratus captured we found only Paratrichobius longicrus (Miranda-Ribeiro, 1907) with prevalence of $32 \%$ and mean intensity of 1.50 .

KEY WORDS. Ectoparasites; Phyllostomidae; prevalence.
\end{abstract}

RESUMO. Informações sobre estreblídeos e nicteribiídeos e seus hospedeiros são escassas na Floresta com Araucária (FA), não havendo dados sobre a riqueza, o padrão de distribuição e a abundância desses ectoparasitos sobre uma determinada comunidade de morcegos. $\mathrm{O}$ presente estudo foi desenvolvido em fragmentos de FA (aproximadamente 470 ha) localizados no município de Fazenda Rio Grande, Paraná, no período de dezembro de 2001 a maio de 2003. Foram registrados 12 indivíduos pertencentes a três espécies de Nycteribiidae e 119 de seis espécies de Streblidae sobre 127 hospedeiros examinados (quatro espécies de Phyllostomidae e sete de Vespertilionidae). Cinco das seis espécies de estreblídeos foram encontradas apenas sobre Sturnira lilium (E. Geoffroy, 1810) e Artibeus lituratus (Olfers, 1818). Em 62 indivíduos de S. lilium foram coletadas: Megistopoda proxima (Séguy, 1926) com prevalência de 65,4\% e intensidade média de infestação de 2,00; Aspidoptera falcata Wenzel, 1976 com 13,5\% e 2,29; Trichobius phyllostomae Kessel, 1925 com 7,7\% e 1,00; e Metelasmus wenzeli Graciolli \& Dick, 2004 com 1,6\% e 4,00. Não foi encontrada diferença na prevalência ( $\mathrm{p}=$ $0,544)$ e na intensidade média $(p=0,361)$ em relação ao sexo de $S$. lilium. Em 26 A. lituratus, foi encontrada apenas Paratrichobius longicrus (Miranda-Ribeiro, 1907) com prevalência de 32\% e intensidade média de 1,50.

PALAVRAS-CHAVE. Ectoparasito; Phyllostomidae; prevalência.

Originalmente, a Floresta com Araucária, também denominada Floresta Ombrófila Mista (FOM), distribuía-se de forma contínua nos estados do Paraná, Santa Catarina e Rio Grande do Sul, tendo como ponto mais setentrional de ocorrência a fronteira entre Minas Gerais e Espírito Santo, na Serra do Caparaó (Hueck 1972, Veloso et al. 1992).

No Paraná, esta formação florestal já representou cerca de 37\% da área do estado, com aproximadamente 75 mil km²
(MACHADO \& SiqueIRA 1980, MAACK 1981); hoje, porém, restaram menos de $1 \%$ de florestas em estágio avançado de sucessão, distribuídas nas mais diversas situações ambientais e em relação ao uso da terra (CASTELA \& BRITEZ 2004).

Segundo MIRETZKı (2003), esta é a segunda fitofisionomia paranaense em número de espécies de morcegos, abrigando 36 das 53 registradas para o estado. Associadas sobre estes hospedeiros foram observadas 25 espécies de dípteros ectoparasitos, 
das quais sete de Basilia Miranda-Ribeiro, 1903 (Nycteribiidae) e 18 de Streblidae (Prevedello et al. 2005). Embora a FOM do Paraná possua uma grande riqueza de quirópteros, tendo também a maior riqueza de dípteros, não há informações sobre a diversidade e o padrão de distribuição desse grupo sobre uma determinada comunidade de morcegos. O objetivo deste trabalho é descrever a comunidade de dípteros ectoparasitos de morcegos em fragmentos de Floresta com Araucária no sudeste do estado do Paraná, sul do Brasil.

\section{MATERIAL E MÉTODOS}

A área de estudo, Fazenda Experimental Gralha Azul, pertencente à Pontifícia Universidade Católica do Paraná e está localizada no município de Fazenda Rio Grande (região metropolitana de Curitiba), sudeste do estado do Paraná, sul do Brasil, entre 2537'-2541'S e 4915'-4917'W. O clima da região é classificado como mesotérmico subtropical úmido (Cfb de Köppen), com verões frescos, sem estação seca definida e com geadas severas e freqüentes (IAPAR 1978, MAACK 1981). A temperatura média dos meses mais quentes é inferior a $22^{\circ} \mathrm{C}$ e a dos meses mais frios é inferior a 18ㄷ (FerReIRA 1996). Os índices pluviométricos anuais variam de 1300 a $1400 \mathrm{~mm}$ e a umidade relativa do ar apresenta índices médios entre 80 e 85\% (IAPAR 1978). A paisagem local é representada por um mosaico formado por fases sucessionais distintas da FOM , sob variados níveis de antropismo (Koenler et al. 1998). Os fragmentos florestais amostrados totalizam cerca de 470 ha (coordenadas: 25039'S-4916'W).

No período de dezembro de 2001 a maio de 2003, foram realizadas capturas mensais de morcegos com redes-de-neblina, num esforço amostral total de $73788 \mathrm{~m}^{2}$.h, valor calculado de acordo com StRAUBE \& BiAnconı (2002) (i.e. multiplicando a área de cada rede pelo tempo de exposição, multiplicado pelo número de repetições e, por fim, pelo número de redes). Adicionalmente, al guns exemplares foram obtidos no sótão de residência localizada próxima ao remanescente de floresta, com auxílio de um puçá.

Os morcegos capturados foram revisados visualmente e os parasitos coletados com o auxílio de pinças ou manualmente, sendo fixados em álcool 70\% para posterior identificação. 0 ordenamento taxonômico dos quirópteros seguiu o proposto por Simmons (2005) e o de Streblidae e Nycteribiidae por GuerRero (1997) e THEOdor (1967) e GueRrero (1996), respectivamente.

\section{RESULTADOS E DISCUSSÃO}

Foram coletados 12 indivíduos pertencentes a três espécies de Nycteribiidae e 119 de seis espécies de Streblidae sobre 127 morcegos examinados, sendo quatro espécies de Phyllostomidae e sete de Vespertilionidae (Tab. I). Todas as espécies de hospedeiros e de parasitos já tin ham sido anteriormente registradas na FOM do Paraná (M IRetzkı 2003, Prevedello et al. 2005), representando 30,5\% das espécies de morcegos e 36,0\% de dípteros ectoparasitos na fitofisionomia. Na Região Neotropical, as comunidades de morcegos são normal mente compostas predominantemente por morcegos filostomídeos (Fenton et al. 1992, Simmons \& Voss 1998, BiAnconi et al. 2004), ao contrário da área de estudo, onde quase 2/3 das espécies pertencem à

Tabela I. Morcegos e seus ectoparasitos encontrados em áreas de Floresta com Araucária da Fazenda Gralha Azul, município de Fazenda Rio Grande, Paraná, durante dezembro de 2001 a outubro de 2002. (Str) Streblidae, (Nyc) Nycteribiidae.

\begin{tabular}{|c|c|c|c|}
\hline Morcego & $\mathrm{N}$ & Díptero & $\mathrm{N}$ \\
\hline \multicolumn{4}{|l|}{ Phyllostomidae } \\
\hline Desmodus rotundus (E. Geoffroy, 1810) & 11 & - & - \\
\hline Artibeus lituratus (Olfers, 1818) & 26 & Paratrichobius longicrus (Miranda-Ribeiro, 1907) (Str) & 12 \\
\hline \multirow[t]{4}{*}{ Sturnira lilium (E. Geoffroy, 1810) } & 52 & Trichobius phyllostomae Kessel, 1925 (Str) & 4 \\
\hline & & Megistopoda proxima (Séguy, 1926) (Str) & 78 \\
\hline & & Aspidoptera falcata Wenzel, 1976 (Str) & 16 \\
\hline & & M etelasmus wenzeli Graciolli \& Dick, 2004 (Str) & 4 \\
\hline Pygoderma bilobatum (Wagner, 1843) & 19 & - & - \\
\hline \multicolumn{4}{|l|}{ Vespertilionidae } \\
\hline Eptesicus brasiliensis (Desmarest, 1819) & 1 & Anatrichobius passosi Graciolli, 2003 (Str) & 1 \\
\hline E. furinalis (d'Orbygny, 1847) & 1 & - & - \\
\hline Lasiurus blossevillii (Lesson \& Garnot, 1826) & 1 & - & - \\
\hline Histiotus velatus (I. Geoffroy, 1824) & 5 & Basilia plaumanni Scott, 1936 (Nyc) & 4 \\
\hline M yotis levis (I. Geoffroy, 1824) & 2 & - & - \\
\hline M. nigricans (Schinz, 1821) & 3 & Basilia andersoni Peterson \& Maa, 1970 (Nyc) & 7 \\
\hline \multirow[t]{2}{*}{ M. ruber (E. Geoffroy, 1806) } & 6 & Basilia ruiae Graciolli, 2003 (Nyc) & 1 \\
\hline & & Anatrichobius passosi Graciolli, 2003 (Str) & 4 \\
\hline Total 11 espécies & 127 & 9 espécies & 131 \\
\hline
\end{tabular}

Revista Brasileira de Zoologia 24 (1): 246-249, março 2007 
Tabela II. Prevalência e intensidade média de infestação (intervalo de confiança de 95\%) de moscas estréblidas sobre Sturnira lilium e Artibeus lituratus na Fazenda Gralha Azul, município de Fazenda Rio Grande, Paraná.

\begin{tabular}{lccccc}
\hline \multirow{2}{*}{ Índice } & \multicolumn{3}{c}{ Sturnira lilum } & & Artibeus lituratus \\
\cline { 2 - 5 } \cline { 2 - 5 } & A. falcata & M. proxima & M. wenzeli & T. phyllostomae & P. longicrus \\
\hline $\mathrm{P}(\%)$ & 13,5 & 65,4 & 1,6 & 7,7 & 32,0 \\
IM (IC) & $2,29(1,57-3,43)$ & $2,00(1,71-2,26)$ & $4,00(-)$ & $1,00(-)$ & $1,50(1,00-2,50)$ \\
\hline
\end{tabular}

família Vespertilionidae. Sabe-se que há uma correlação positiva entre a riqueza de estreblídeos e a de morcegos, especialmente da família Phyllostomidae (Dıck \& Getrınger 2005). Corroborando esta informação, em áreas de Floresta Atlântica (s.s.) e Cerrado, com maior número de espécies de morcegos filostomídeos também foi registrado maior número de espécies de estreblídeos. Na Mata Atlântica nos estados de Minas Gerais (Azevedo \& Linardi 2002), São Paulo (Bertola et al. 2005) e Rio Grande do Sul (RuI \& Graciolu 2005), foram encontradas respectivamente, oito, 11 e sete espécies de estreblídeos sobre oito, 11 e 14 espécies de morcegos filostomídeos. No Cerrado, KOMENO \& LINHARES (1999) registraram 11 espécies de estreblídeos sobre 12 espécies de morcegos filostomídeos. Sendo assim, a menor riqueza de estreblídeos encontrada na Fazenda Gralha Azul pode ser explicada pela baixa riqueza de Phyllostomidae.

Entre as associações parasito-hospedeiro encontradas, Anatrichobius passosi Graciolli, 2003 é registrada pela primeira vez sobre Eptesicus brasiliensis (Desmarest, 1819). Este registro pode representar uma baixa especificidade deste estreblídeo, o qual anteriormente tinha sido registrado apenas sobre morcegos do gênero Myotis Kaup, 1826 (Graciolli 2003, Bertola et al. 2005), ou uma ocorrência temporária ou acidental. Comparando com os esparsos dados quantitativos de dípteros ectoparasitos sobre morcegos vespertilionídeos, BERTOLA et al. (2005) encontraram A. passosi somente sobre as espécies de Myotis.

Na tabela II são mostrados os valores de prevalência e intensidade média de infestação dos estreblídeos encontrados sobre Sturnira lilium (E. Geoffroy, 1810) e Artibeus lituratus (Olfers, 1818). A comparação destes resultados foi restringida apenas a trabal hos com período de coleta similar e realizados numa única localidade (Komeno \& LinAREs 1999, Bertola et al. 2005, RuI \& Graciolli 2005). Assim, até o momento os valores de intensidade média igual ou acima de dois estão entre os maiores encontrados para estas espécies de estreblídeos. Sobre S. Iilium em área de Cerrado a intensi dade média foi de 1,6 para Aspidoptera falcata Wenzel, 1976 e 1,8 para Megistopoda proxima (Séguy, 1926) (Komeno \& Linhares 1999) e na Floresta Atlântica foi de 1,04 e 2,17 para M. proxima (Bertola et al. 2005, Rui \& Graciolli 2005, respectivamente) e 1,67 para Trichobius phyllostomae Kessel, 1925 (Ruı \& Gracıoll 2005). Em relação a A. lituratus na Mata Atlântica, a intensidade de Paratrichobius Iongicrus (Miranda-Ribeiro, 1907) foi de 1,82 e 1,68 (Bertola et al. 2005, RuI \& GraciolLI 2005, respectivamente). Os valores de prevalência podem indicar que a distribuição de estreblídeos sobre diferentes populações de hospedeiros é bastante variável.

Revista Brasileira de Zoologia 24 (1): 246-249, março 2007
Em áreas de Mata Atlântica, a prevalência de M. proxima sobre S. lilium é abaixo de $30 \%$, enquanto na Fazenda Gral ha Azul superior a $60 \%$. Trichobius phyllostomae é encontrada apenas na Floresta com Araucária e Mata Atlântica (s.s.), dois biomas ameaçados de extinção, nos estados de São Paulo, Paraná, Santa Catarina e Rio Grande do Sul, em prevalência inferior a 10\% (Ruı \& Graciolli 2005, neste trabalho), embora seu único hospedeiro tenha ampla distribuição geográfica (Simmons 2005). Da mesma forma, M etelasmus wenzeli Graciolli \& Dick, 2004 também parece ocorrer em baixas densidades. Dick \& GetTINGER (2005) registraram prevalência e intensidade média inferiores a $1 \%$ e 2 , respectivamente de $M$. wenzeli sobre 406 indivíduos de S. Iilium examinados de várias localidades no Paraguai. A contínua destruição de hábitats associada à baixa densidade populacional dessas duas espécies de estreblídeos, provavelmente colocará ambas em ameaça de extinção.

Não foi encontrada diferença na prevalência $\left(\chi^{2}=0,789\right.$, $\mathrm{df}=1, p=0.544$ ) e na intensidade média ( $t=0.930, p=0,361)$ de estreblídeos em relação ao sexo de S. lilium (Tab. III). Relação similar também havia sido encontrada por Ruı \& Gracıollı (2005).

Como visto neste trabalho, o número de espécies de dípteros ectoparasitos de morcegos e seus hospedeiros na Fazenda Gralha Azul representam cerca de 1/3 das espécies

Tabela III. Prevalência (P) (\%) e intensidade média de infestação (IM), com respectivo intervalo de confiança de $95 \%$, de Streblidae sobre machos e fêmeas de Sturnira lilium na Fazenda Gralha Azul, município de Fazenda Rio Grande, Paraná.

\begin{tabular}{lcc}
\hline \multicolumn{1}{c}{ Índice } & Macho & Fêmea \\
\hline $\mathrm{P}(\%)$ & 60,0 & 71,9 \\
IM (IC) & $2,17(1,58-2,67)$ & $2,65(2,04-3,65)$ \\
\hline
\end{tabular}

registradas na Floresta com Araucária. Este resultado ressalta a importância de novos estudos em outros locais, principalmente em áreas protegidas, para que haja uma maior compreensão da estrutura das comunidades e da distribuição de estreblídeos e nicteribiídeos em toda a fitofisionomia.

\section{AGRADECIMENTOS}

Ao Programa Ecológico de Longa Duração - PELD/CNPq (Site 9) pelo auxílio financeiro; aos funcionários e administradores da Fazenda Experimental Gralha Azul (PUCPR); a Sylvio Péllico Netto; à Fabiana R. Mendes, Rodrigo P. Napoli e Daniel 
C. Carneiro pelo apoio em campo; e à Sandra B. Mikich pela confecção do abstract.

\section{REFERÊNCIAS BIBLIOGRÁFICAS}

Azevedo, A.A. \& P.M. LinARDi. 2002. Streblidae (Diptera) of phyllostomid bats from Minas Gerais, Brazil. Memórias do Instituto Oswaldo Cruz 97: 421-422.

Bertola, P.B; C.C. Aires; S.E. Favorito; G. Graciolli; M. Amaku \& R. Pinto-da-Rocha. 2005. Bat flies (Diptera: Streblidae, Nycteribiidae) parasitic on bats (Mammalia: Chiroptera) at Parque Estadual da Cantareira, São Paulo, Brazil: parasitism rates and host-parasite associations. Memórias do Instituto Oswaldo Cruz 100: 25-32.

Bianconı, G.V.; S.B. MIKICH \& W.A. Pedro. 2004. Diversidade de morcegos (Mammalia, Chiroptera) em remanescentes florestais do município de Fênix, noroeste do Paraná, Brasil. Revista Brasileira de Zoologia 21 (4): 943-954.

CASTELLA, P.R. \& R.M. BRITEZ. 2004. A floresta com araucária no Paraná: conservação e diagnóstico dos remanescentes florestais. Brasília, Fundação de Pesquisas Florestais do Paraná. Fundação de Pesquisas Florestais do Paraná, 236p.

Dick, C.W. \& D. Gettinger. 2005. A faunal survey of streblid flies (Diptera: Streblidae) associated with bats in Paraguay. Journal of Parasitology 91: 1015-1024.

Fenton, M.B.; L. Acharya; D. Audet; M.B.C. Hickey; C. Merriman; M.K. OвRIst \& D.M. SYme. 1992. Phyllostomid bats (Chiroptera: Phyllostomidae) as indicators of habitat disruption in the Neotropics. Biotropica 24 (3): 440-446.

Ferreira, J.C.V. 1996. O Paraná e seus municípios. Maringá, Memória Brasileira, 728p.

Graciolli, G. 2003. Nova espécie de Anatrichobius Wenzel, 1966 (Diptera, Streblidae) do Brasil meridional. Revista Brasileira de Entomologia 47: 55-58.

Guerrero, R. 1996. Streblidae (Diptera: Pupipara) parasitos de Ios murciélagos de Pakitza, Parque Nacional Manu (Perú), p. 627-641. In: D. E. Wilson \& A. SAndoval (Eds). MANU: La biodiversidad del sureste del Perú. Washington, Smithsonian Institution, 679p.

Guerrero, R. 1997. Catalogo de los Streblidae (Diptera: Pupipara) parásitos de murciélagos (Mammalia: Chiroptera) del Nuevo Mundo. VII. Lista de especies, hospedadores y paises. Acta Biologica Venezuelana 17: 9-24.

HUECK, K. 1972. Asflorestas da América do Sul: ecologia, composição e importância econômica. São Paulo, Editora Polígono, 466p.

IAPAR. 1978. Cartas climáticas básicas do Estado do Paraná. Curitiba, Instituto Agronômico do Paraná, 41p.

Koehler, A.; S.N. Péllico \& C.R. Sanquetta. 1998. Análise da estrutura de uma Floresta Ombrófila Mista semi devastada, Fazenda Gral ha Azul, região Metropolitana de Curitiba, com implicações ao manejo. Revista Acadêmica 9 (1): 37-60.

Komeno, C.A. \& A.X. Linhares. 1999. Batflies parasitic on some phyllostomid bats in Southeastern Brazil: parasitism rates and host-parasite relationships. Memórias do Instituto Oswaldo Cruz 94 94: 151-156.

MAACK, R. 1981. Geografia física do Estado do Paraná. Rio de Janeiro, José Olympio, Secretaria da Cultura e do Esporte do Governo do Estado do Paraná, XLIII+442p.

Machado, S.A. \& J.D.P. SiquelRA. 1980. Distribuição natural da Araucaria angustifolia (Bert.) O Ktze., p.4-9. In: IUFRO Meeting on Forestry Problems of the Genus Araucaria. Forestry problems of the genus Araucaria. Curitiba, FUPEF, $X V I I I+382 p$.

MIRETZKı, M. 2003. Morcegos do estado do Paraná, Brasil (Mammalia, Chiroptera): riqueza de espécies, distribuição e síntese do conhecimento atual. Papéis Avulsos de Zoologia 43: 101138.

Prevedello, J.A.; G. Graciolli \& C.J.B. de Carvalho. 2005. A fauna de dípteros (Streblidae e Nycteribiidae) ectoparasitos de morcegos (Chiroptera) do estado do Paraná, Brasil: composição, distribuição e áreas prioritárias para novos estudos. Biociências 13: 193-209.

RuI, A.M. \& G. Graciollı. 2005. Moscas ectoparasitas (Diptera, Streblidae) de morcegos (Chiroptera, Phyllostomidae) no sul do Brasil: associações hospedeiros-parasitos e taxas de infestação. Revista Brasileira de Zoologia 22: 438-445.

Simmons, N.B. 2005. Order Chiroptera, p. 312-529. In: D.E. WILSON \& D.M. REEDER (Eds). Mammal species of the World: a taxonomic and geographic reference. Baltimore, Maryland, Johns Hopkins University Press, $3^{\text {rd }}$ ed., 2142p.

Simmons, N.B. \& R.S. Voss. 1998. The mammals of Paracou, French Guiana: a Neotropical lowdland rainforest fauna. Part 1. Bats. Bulletin of the American Museum of Natural History 237: 1-219.

Straube, F.C. \& G.V. Bianconı. 2002. Sobre a grandeza e a unidade utilizada para estimar esforço de captura com utilização de redes-de-neblina. Chiroptera Neotropical 8 (1-2): 150152.

TheOdoR, O. 1967. An illustrated catalogue of the Rothschild collection of Nycteribiidae (Diptera) in the British Museum (Natural History) with keys and short descriptions for the identification of subfamilies, genera, species and subspecies. British Museum (Natural History) Publication 665: 1-506.

Veloso, H.P.; L.C. Oliveira-Filho; A.M.S.F. Vaz; M.P.M. Lima; R. Marquete \& J.E.M. BrazÃo. 1992. Manual técnico da vegetação brasileira. Manuais técnicos em geociências. Rio de Janeiro, Secretaria de Planejamento, Orçamento e Coordenação, Fundação Instituto Brasileiro de Geografia e Estatística, Diretoria de Geociências, Departamento de Recursos Naturais e Estudos Ambientais, 93p.

Recebido em 10.X.2006; aceito em 09.III.2007.

Revista Brasileira de Zoologia 24 (1): 246-249, março 2007 